\title{
Repelencia e inhibición de oviposición de Diaphorina citri (Hemiptera: Liviidae) con aceites minerales en condiciones controladas
}

\author{
Repellence and oviposition inhibition of Diaphorina citri (Hemiptera: Liviidae) with \\ minerals oils in controlled conditions
}

\author{
(iD LAURA DELIA ORTEGA-ARENAS ${ }^{1 *}$, iD MARIO APÁEZ-BARRIOS ${ }^{1}$, \\ (iD JORGE MANUEL VALDEZ-CARRASCO ${ }^{1}$ \\ (iD JUAN FERNANDO SOLÍS-AGUILAR ${ }^{2}$
}

${ }^{1}$ Colegio de Postgraduados, Texcoco, México.ladeorar@colpos.mx, apaez.mario@colpos.mx, jvaldez@colpos.mx

${ }^{2}$ Universidad Autónoma Chapingo, Chapingo, México.jfsolis@hotmail.com

\section{Autor de correspondencia}

Laura Delia Ortega-Arenas. Campus Montecillo, Colegio de Postgraduados, Montecillo, Texcoco, Estado de México, 56230, México. ladeorar@colpos.mx

\section{Citación sugerida}

ORTEGA-ARENAS, L. D.; APÁEZ-BARRIOS, M.; VALDEZ-CARRASCO, J. M.; SOLIS-AGUILAR, J. F. 2022. Repelencia e inhibición de oviposición de Diaphorina citri (Hemiptera: Liviidae) con aceites minerales en condiciones controladas. Revista Colombiana de Entomología 48 (1): e11272. https://doi. org/10.25100/socolen.v48i1.11272

Recibido: 13-May-2021

Aceptado: 18-Ene-2022

Publicado: 04-Mar-2022

Revista Colombiana de Entomología ISSN (Print): 0120-0488

ISSN (On Line): 2665-4385

https://revistacolombianaentomologia.univalle.edu.co

Open access

(c) (1) (2) (2) BY-NC-SA 4.0

Publishers: Sociedad Colomsiana de Entomologia

Publishers: Sociedad Colombiana de Entomología SOCOLEN (Bogotá, D. C., Colombia) https://www.socolen.org.co

Universidad del Valle (Cali, Colombia)

https://www.univalle.edu.co

(C) 2021 Sociedad Colombiana de Entomología SOCOLEN y Universidad del Valle - Univalle
Resumen: Diaphorina citri es una de las plagas más importantes de cítricos debido a que es el vector de Candidatus Liberibacter asiaticus, agente causal del Huanglongbing (HLB) en cítricos. Su manejo se basa en el uso de insecticidas y recientemente se ha explorado el uso de productos alternativos de manejo, como los aceites minerales, por lo que el objetivo del presente trabajo fue evaluar, en condiciones controladas, la actividad repelente e inhibitoria de la oviposición de los aceites minerales Akaroil ${ }^{\circledR}$, Anasef-T $\AA$, Saf-T-Side $\AA$ y Stylet Oil $®$ en $D$. citri. La repelencia se determinó al exponer adultos a una hoja de naranja var. Valencia previamente tratada y la inhibición de la oviposición por el método de aplicación residual. Los cuatro aceites minerales causaron actividad repelente significativa $\geq 90 \%$ a $35 \mathrm{mg} / \mathrm{mL}$, no obstante, ésta disminuyó a concentraciones por debajo de $10 \mathrm{mg} / \mathrm{mL}$. Como repelente sobresalió el aceite Saf-T-Side $\left(\mathrm{CR}_{50}=0,14 \mathrm{mg} / \mathrm{mL}\right)$, seguido de Anasef-T, Akaroil y Stylet-Oil, con $\mathrm{CR}_{50}$ de $1,33,1,92$ y $3,32 \mathrm{mg} / \mathrm{mL}$, respectivamente, a las $24 \mathrm{~h}$. dosis de $1 \mathrm{mg} / \mathrm{mL}$. Con todos los aceites, se inhibió hasta en $90 \%$ la oviposición. La actividad de los aceites minerales debe validarse en condiciones de campo para determinar el potencial real de esta alternativa en el manejo integrado de $D$. citri.

Palabras clave: Actividad biológica, HLB, manejo integrado de plagas, plagas de cítricos, plaguicidas biorracionales, psílido asiático de los cítricos

Abstract: Diaphorina citri is one of the most important pests in citrus orchards and is associated with the transmission of Huanglongbing (HLB), a disease caused by the bacterium Candidatus Liberibacter asiaticus on citrus. Currently, the use of chemical insecticides is the most common control strategy for controlling $D$. citri; however, other alternatives such as the use of mineral oils have been considered. Based on this, we studied the repellence and oviposition inhibition activity by the commercial mineral oils Akaroil ${ }^{\circledR}$, Anasef - T $®$, Saf-T - Side ${ }^{\circledR}$ and Stylet Oil ${ }^{\circledR}$ on $D$. citri. The repellency was determined by exposing adults to previously treated orange leaves with different concentrations of mineral oils and the inhibition of oviposition by the residual application method. All tested oils caused significant $\geq 90 \%$ repellent activity to $35 \mathrm{mg} / \mathrm{mL}$, however, this decreased to concentrations below $10 \mathrm{mg} / \mathrm{mL}$. The greatest repellent activity was achieved by the Saf-T-Side oil $\left(\mathrm{RC}_{50}=0.14 \mathrm{mg} / \mathrm{mL}\right)$, followed by Anasef-T, Akaroil and Stylet Oil, with $\mathrm{CR}_{50}$ of $1.33,1.92,3.32 \mathrm{mg} / \mathrm{mL}$, respectively. Doses as low as 1 $\mathrm{mg} / \mathrm{mL}$ were enough to inhibit oviposition in up to $90 \%$ in all tested oils. We conclude that the activity of mineral oils should be validated under field conditions to determine if they represent a useful tool within $D$. citri integrated management.

Keywords: Asian citrus psyllid, biological activity, biorational pesticides, citrus pests, HLB, integrated pest management.

\section{Introducción}

El Psílido Asiático de los Cítricos (PAC) Diaphorina citri Kuwayama, 1908 (Hemiptera: Liviidae) es el vector primario de la bacteria Candidatus Liberibacter, causante de la enfermedad más devastadora en cítricos en el mundo conocida como Huanglongbing (HLB) (Da Graça et al. 2016; Olvera-Vargas et al. 2020). El manejo 
integrado del PAC-HLB se lleva a cabo mediante el empleo de varias prácticas como el uso de plantas certificadas, eliminación de árboles enfermos y control del vector (Mora-Aguilera et al. 2014; SENASICA 2020). El control del vector es la estrategia predominante y se realiza por medio del control químico con insecticidas de diferentes grupos toxicológicos (García-Méndez et al. 2016; SENASICA 2020), práctica que ha repercutido, en algunos casos, en la creación de poblaciones resistentes a insecticidas (Tiwari et al. 2012; García-Méndez et al. 2016; Pérez-Zarate et al. 2016; García-Méndez et al . 2018), contaminación ambiental e impacto en la salud humana (Del Puerto et al. 2014).

Además del uso de insecticidas como principal estrategia de control se han explorado otros productos para el manejo de plagas en diversos cultivos, incluyendo cítricos, como aceites derivados del petróleo con acción insecticida (Martínez-Ferrer et al. 2003; Varela et al. 2004; Chen et al. 2009; Hoy 2011). Diversos estudios reportan que los aceites minerales son seguros a enemigos naturales, no promueven el desarrollo de resistencia, presentan baja toxicidad a vertebrados y su degradación es rápida en el ambiente (Beattie et al. 1995; Rae et al. 1997). Específicamente en cítricos, los aceites muestran efecto tóxico y repelente en el PAC (Leong et al. 2012; Yang et al. 2013) e inhibitorio de la emergencia y alimentación del psílido (Hu et al. 2020). El efecto tóxico se asocia con la interacción entre las propiedades físico-químicas del aceite y las características anatómicas, fisiológicas y de comportamiento del insecto (Nguyen et al. 2007; Najar-Rodríguez et al. 2008; Stadler y Buteler 2009; Buteler y Stadler 2011), provocando asfixia (Kallianpur et al. 2002; Taverner 2002), ruptura del integumento, alteración de la actividad enzimática y hormonal (Smith y Salkeld 1966; Sieburth et al. 1998; Stadler y Buteler 2009; Buteler y Stadler 2011). En cambio, la repelencia se relaciona con la supresión de volátiles liberados por las plantas al formar una fina película sobre la superficie foliar y el efecto directo de los compuestos liberados por la degradación del aceite (Stansly et al. 2002; Ouyang et al. 2008, 2013), además de la inhabilitación de sitios de alimentación y oviposición (Hu et al. 2020). En México se promueve el uso de aceites minerales para el combate de PAC en campo, ya sea empleándolos solos, como coadyuvantes y/o en combinación con insecticidas convencionales (SENASICA 2020), lo que implica cierto riesgo al propiciar una mayor presión de selección al aumentar la persistencia de los insecticidas convencionales (Hernández-Fuentes et al. 2012; Pérez-Zarate et al. 2016). Por ello la tendencia actual en la protección de cultivos se basa en la reducción y sustitución de los productos más tóxicos por otros respetuosos con el medio ambiente y fauna benéfica, y es en este contexto que los aceites minerales podrían jugar un papel importante. Por lo que se planteó como objetivo evaluar el efecto repelente e inhibidor de la oviposición de cuatro aceites minerales sobre adultos de PAC, en condiciones controladas, con el fin de encontrar productos alternativos para el manejo integrado de $D$. citri.

\section{Materiales y métodos}

La investigación se realizó de septiembre a febrero de 2017 2018 , bajo condiciones controladas, en el laboratorio e invernadero de Insectos Vectores del Colegio de Postgraduados, Campus Montecillo, Texcoco, Estado de México, México.

Cría de Diaphorina citri. Se utilizaron individuos de una colonia de D. citri existente en el área de Insectos Vectores, la cual se mantiene desde el 2009, con aproximadamente 1000 adultos (proporción sexual 1 $\delta^{\hat{2}}: 1$ 우) que se recolectaron en follaje de naranja Citrus sinensis (L.) Osbeck cv. Valencia (Rutaceae) y plantas de limonaria Murraya paniculata (L.) Jack (Rutaceae) en la comunidad de Cazones de Herrera, Veracruz, México, misma que se mantuvo bajo cría continua y aislada en invernadero. Para incrementar la colonia, los adultos se introdujeron en jaulas entomológicas $(60 \times 40 \times 60 \mathrm{~cm}) \mathrm{cu}-$ biertas con tela de organza, donde se colocaron, de manera simultánea, plantas de limonaria y naranja de $120 \mathrm{~d}$ de edad, en bolsas de plástico de $5 \mathrm{~L}$ que contenían una mezcla de vermicomposta, tierra de hoja y vermiculita $(3: 2: 1)$ como medio de soporte y nutrición. Los adultos se mantuvieron sobre las plantas durante una semana para que ovipositaran, después se retiraron con un aspirador bucal y las plantas infestadas se mantuvieron en condiciones de invernadero $\left(27 \pm 5{ }^{\circ} \mathrm{C}\right.$, HR $60 \pm 5 \%$ y $12 \mathrm{~h}$ de fotoperiodo) hasta la emergencia de los nuevos adultos; proceso que se realizó periódicamente para mantener alta población de cría y para contar con material biológico durante el experimento.

Aceites minerales evaluados y preparación. Se evaluaron cuatro aceites minerales de uso común para el control de D. citri en las principales zonas productoras de cítricos en México (SENASICA 2020). Se evaluaron los productos: Akaroil ${ }^{\circledR}$ (Velsimex, Ciudad de México, México, aceite mineral $98,5 \%$, residuo insulfonable (UR $=92 \%$ ); Anasef-T ${ }^{\circledR}$ (Agricultura Nacional de Jalisco, Jalisco, México, aceite parafínico de petróleo $80 \%$, (UR no disponible); Saf-T-Side ${ }^{\circledR}$ (Distribuciones Imex, Jalisco, México, aceite parafínico de petróleo $80 \%$, (UR $=92 \%$ ) y Stylet Oil ${ }^{\circledR}$ (Gowan Mexicana, Baja California, México, aceite mineral 97,1\% (UR = $99 \%)$. Los productos se diluyeron en agua destilada con Inex-A ${ }^{\circledR}($ Cosmocel, Nuevo León, México, alcohol graso etoxilado 20,2 \%) al $0,1 \%$ como adherente.

Bioensayo de repelencia. La repelencia se evaluó con el método de cilindro (olfatómetro) propuesto por Schuster et al. (2009) con ligeras modificaciones. Para ello se construyó una arena experimental conformada por un vaso desechable de polipropileno (Cristal ${ }^{\circledR}$ Bosco, México; de $250 \mathrm{~mL}$ ), con tres orificios; dos laterales (Ø $4.0 \mathrm{~cm})$ cubiertos por una malla fina para permitir la ventilación y uno frontal medio $(\varnothing 0,8$ $\mathrm{cm})$ con un tapón extraíble de corcho. La tapa del vaso se perforó para acoplar un vial de cristal de $5 \mathrm{~mL}$ con agua de la llave, en el cual se sumergió y sujetó el peciolo de una hoja de naranja $C$. sinensis previamente inmersa por $5 \mathrm{~s}$ en los aceites minerales. El vial y hoja se encerraron acoplando la tapa con el vaso en posición invertida. Por último, por el orificio medio, se introdujeron 20 adultos (proporción sexual $1 \delta^{\hat{A}}: 1$ ) de 3 a $6 \mathrm{~d}$ de emergidos y $2 \mathrm{~h}$ en ayuno previo. Las arenas experimentales se mantuvieron a $27 \pm 5{ }^{\circ} \mathrm{C}$, HR $60 \pm 5 \%$ y $12 \mathrm{~h}$ de fotoperiodo.

La repelencia se midió por la diferencia entre insectos posados y no posados en la hoja testigo comparada con las hojas tratadas a las 4, 5, 6 y 24 h después de la introducción de los insectos, y se expresó en porcentaje ( 20 adultos repelidos $=$ $100 \%$ repelencia en cada repetición). El porcentaje de repelencia aceptable para el testigo fue $\leq 12 \%$ (Robertson et al. 2007).

Bioensayo de inhibición de oviposición. Para llevar a cabo esta prueba se utilizó la misma arena experimental empleada en los ensayos de repelencia con ligeras modificacio- 
nes. En lugar de una hoja se seleccionaron brotes vegetativos de naranja $C$. sinensis mismos que se sumergieron por $5 \mathrm{~s}$ en los aceites minerales y dejaron secar a temperatura ambiente durante $30 \mathrm{~min}$. El vial y brote se encerraron acoplando la tapa con el vaso en posición invertida. Por último, por el orificio medio, se introdujeron 20 adultos (proporción sexual $1 \delta^{\Uparrow}: 1$ + $)$ de 3 a 6 días de emergidos y $2 \mathrm{~h}$ en ayuno previo. Las arenas experimentales se mantuvieron a $27 \pm 5{ }^{\circ} \mathrm{C}, \mathrm{HR}$ $60 \pm 5 \%$ y $12 \mathrm{~h}$ de fotoperiodo. Finalmente, transcurridas $48 \mathrm{~h}$, después de la introducción de los psílidos, se realizaron las evaluaciones por medio del conteo de número de huevos desovados en cada uno de los tratamientos con la ayuda de un microscopio estereoscópico. El número de huevos desovados en el testigo se tomó como un $100 \%$.

Selección de dosis a evaluar. Para ambos bioensayos y para cada producto primero se realizó un bioensayo preliminar para determinar la ventana de respuesta biológica donde se utilizaron concentraciones logarítmicas en un rango de 0,00001 a $10 \%$, con el fin de detectar las dosis que ocasionaban repelencia e inhibición de entre 5 y $100 \%$. Después con los resultados obtenidos y para cada producto, se seleccionaron de siete a ocho concentraciones intermedias, en un rango de 0,000001 a $3,5 \%(0,00001$ a $35 \mathrm{mg} / \mathrm{mL})$, para realizar el bioensayo completo. Para cada concentración y repetición siempre se incluyó un testigo con base de agua más Inex- $A \AA$, se trataron 20 adultos de PAC y se realizaron cinco repeticiones en tiempos diferentes. Se utilizó un diseño experimental completamente al azar.

Análisis estadístico. Los datos de repelencia e inhibición de oviposición se corrigieron con la repelencia y oviposición observada en el testigo por medio de la ecuación de Abbott (1925), y se sometieron a un análisis de varianza SAS/STAT 9.1 (SAS 2004), prueba de comparación de medias de Tukey $(\mathrm{p} \leq 0,05)$ y análisis Probit para obtener los valores de la línea de regresión y la concentración repelente media $\left(\mathrm{CR}_{50}\right)$ e inhibitoria media $\left(\mathrm{CIO}_{50}\right)$, que se expresaron en $\mathrm{mg} / \mathrm{mL}$. También se determinó el índice de repelencia (IR) propuesto por Lin et al. (1990), para comparar los efectos de las dosis, este índice se calculó con la fórmula $I R=2 G /(G+P)$, donde $G=$ porcentaje de insectos posados en el tratamiento y $\mathrm{P}=$ porcentaje de insectos posados en el testigo. Los índices se clasificaron como IR $=1$ concentración neutra, IR $<1$ concentración repelente e IR $>1$ concentración atrayente.

\section{Resultados}

Los cuatro aceites minerales evaluados mostraron efecto repelente en todas las horas de evaluación; dicha actividad se relacionó positivamente con la concentración.

La repelencia promedio más alta $(\geq 90 \%)$ para todos los aceites se obtuvo al aplicar la concentración de $35 \mathrm{mg} / \mathrm{mL}$ y el efecto se mantuvo estable $(\mathrm{p} \leq 0,05)$ y en algunos casos se acentuó a través del tiempo (Tabla 1). No se detectaron diferencias significativas $(\mathrm{p} \leq 0,05)$ entre los diferentes tiempos para cada producto a la concentración alta, pero sí entre concentraciones y producto. Para lograr un efecto repelente significativo $(>50 \%$ ) con Akaroil, Anasef-T, y Stylet- Oil, se requirió aplicar concentraciones superiores a $6,0 \mathrm{mg} / \mathrm{mL}$, mientras que el mismo efecto con Saf-T-Side se logró con 0,6 $\mathrm{mg} / \mathrm{mL}$.

Los valores de la concentración repelente media $\left(\mathrm{CR}_{50}\right)$ en los cuatro aceites oscilaron entre 0,14 y $3,78 \mathrm{mg} / \mathrm{mL}$. Destacó Saf-T-Side, con la menor $\mathrm{CR}_{50}=0,24 \mathrm{mg} / \mathrm{mL}$ en promedio en los cuatro tiempos seguido por Anasef-T $(1,61 \mathrm{mg} / \mathrm{mL})$, Akaroil (2,90 mg/mL) y Stylet-Oil (3,34 mg/mL) (Tabla 1). A las $24 \mathrm{~h}$ después de la aplicación se logró la mayor actividad repelente a nivel de $\mathrm{CR}_{50}$ en Saf-T-Side $(0,4 \mathrm{mg} / \mathrm{mL}$ a las 24 h) y Anasef-T (1,33 mg/mLa las $24 \mathrm{~h})$ (Tabla 1). En general actividad repelente $\geq 90 \%$ se observó desde las $3 \mathrm{~h}$ al aplicar la concentración de $35 \mathrm{mg} / \mathrm{mL}$; el efecto se mantuvo estable por varias horas y en algunos casos como en Akaroil y Saf-TSide se incrementó a las $24 \mathrm{~h}$.

El valor de la pendiente para todos los tratamientos osciló entre $0,53 \pm 0,09$ y $0,96 \pm 0,16$ lo cual indica que la población no responde de forma uniforme a la selección con los productos evaluados (Tabla 1). El efecto repelente de cada uno de los productos fue similar y se confirmó con los valores de los IR $<1$ estimados al aplicar concentraciones mayores de $1 \mathrm{mg} /$ $\mathrm{mL}$ de cada uno de los aceites, acción que se mantuvo hasta las $24 \mathrm{~h}$ post aplicación (Tabla 1). Sin embargo, la actividad de los aceites disminuyó en función de la reducción en la concentración.

Tabla 1. Repelencia (\%) de adultos de Diaphorina citri a las 4, 5, 6 y 24 h después de la aplicación de cuatro aceites minerales.

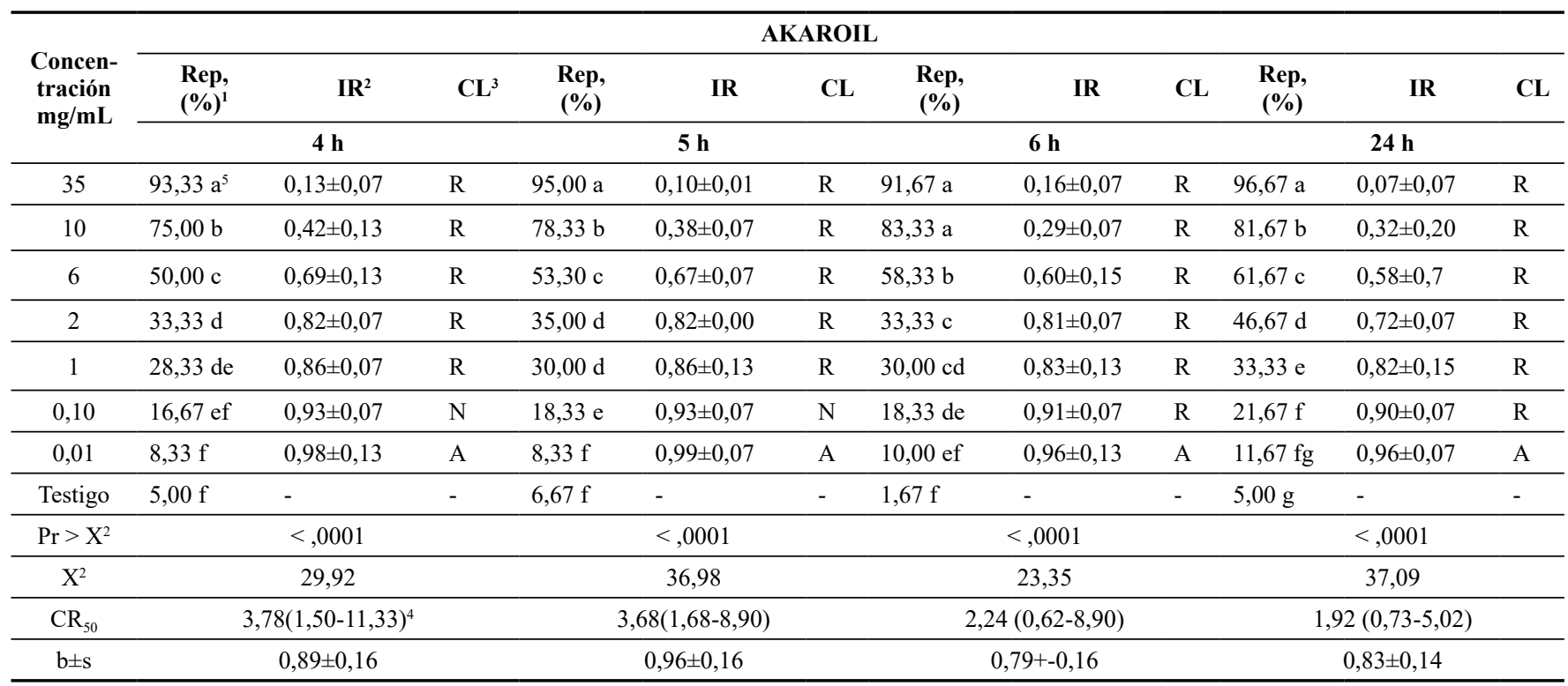




\begin{tabular}{|c|c|c|c|c|c|c|c|c|c|c|c|c|}
\hline \multicolumn{13}{|c|}{ ANASEF-T } \\
\hline 35 & $96,67 \mathrm{a}$ & $0,07 \pm 0,07$ & $\mathrm{R}$ & $96,67 \mathrm{a}$ & $0,07 \pm 0,07$ & $\mathrm{R}$ & $95,00 \mathrm{a}$ & $0,10 \pm 0,13$ & $\mathrm{R}$ & $96,67 \mathrm{a}$ & $0,07 \pm 0,07$ & $\mathrm{R}$ \\
\hline 10 & $85,00 \mathrm{~b}$ & $0,27 \pm 0,00$ & $\mathrm{R}$ & $85,00 \mathrm{~b}$ & $0,27 \pm 0,00$ & $\mathrm{R}$ & $83,33 \mathrm{a}$ & $0,30 \pm 0,07$ & $\mathrm{R}$ & $81,67 \mathrm{~b}$ & $0,32 \pm 0,07$ & $\mathrm{R}$ \\
\hline 6 & $66,67 \mathrm{c}$ & $0,52 \pm 0,15$ & $\mathrm{R}$ & $66,67 \mathrm{c}$ & $0,52 \pm 0,07$ & $\mathrm{R}$ & $63,33 \mathrm{~b}$ & $0,56 \pm 0,07$ & $\mathrm{R}$ & $65,00 \mathrm{c}$ & $0,53 \pm 0,13$ & $\mathrm{R}$ \\
\hline 2 & $51,67 d$ & $0,67 \pm 0,07$ & $\mathrm{R}$ & $50,00 \mathrm{~d}$ & $0,69 \pm 0,13$ & $\mathrm{R}$ & $46,67 d$ & $0,73 \pm 0,07$ & $\mathrm{R}$ & $45,00 \mathrm{~d}$ & $0,73 \pm 0,13$ & $\mathrm{R}$ \\
\hline 1 & $38,33 \mathrm{e}$ & $0,79 \pm 0,07$ & $\mathrm{R}$ & $36,67 \mathrm{e}$ & $0,80 \pm 0,07$ & $\mathrm{R}$ & $33,33 \mathrm{~cd}$ & $0,83 \pm 0,15$ & $\mathrm{R}$ & $33,33 \mathrm{e}$ & $0,82 \pm 0,07$ & $\mathrm{R}$ \\
\hline 0,35 & $31,67 \mathrm{e}$ & $0,84 \pm 0,07$ & $\mathrm{R}$ & $30,00 \mathrm{e}$ & $0,85 \pm 0,00$ & $\mathrm{R}$ & $28,33 \mathrm{~d}$ & $0,87 \pm 0,15$ & A & $26,67 \mathrm{e}$ & $0,86 \pm 0,07$ & $\mathrm{R}$ \\
\hline 0,10 & $30,00 \mathrm{e}$ & $0,85 \pm 0,13$ & $\mathrm{R}$ & $28,33 \mathrm{e}$ & $0,86 \pm 0,07$ & $\mathrm{R}$ & $28,33 \mathrm{~d}$ & $0,87 \pm 0,15$ & A & $26,67 \mathrm{e}$ & $0,86 \pm 0,07$ & $\mathrm{R}$ \\
\hline Testigo & $5,00 \mathrm{f}$ & - & - & $5,00 \mathrm{f}$ & - & - & $6,67 \mathrm{e}$ & - & - & $3,33 \mathrm{f}$ & - & - \\
\hline $\operatorname{Pr}>X^{2}$ & \multicolumn{3}{|c|}{$<, 0001$} & \multicolumn{3}{|c|}{$<, 0001$} & \multicolumn{2}{|r|}{$<, 0001$} & \multicolumn{4}{|c|}{0,0011} \\
\hline $\mathrm{X}^{2}$ & \multicolumn{3}{|c|}{36,57} & \multicolumn{3}{|c|}{38,83} & \multicolumn{2}{|r|}{31,88} & \multicolumn{4}{|c|}{10,58} \\
\hline $\mathrm{CR}_{50}$ & \multicolumn{3}{|c|}{$1,46(0,62-3,18)$} & \multicolumn{3}{|c|}{$1,57(0,70-3,32)$} & \multicolumn{2}{|c|}{$2,11(0,89-5,17)$} & \multicolumn{4}{|c|}{$1,33(0,05-18,05)$} \\
\hline $\mathrm{b} \pm \mathrm{s}$ & \multicolumn{3}{|c|}{$0,86 \pm 0,14$} & \multicolumn{3}{|c|}{$0,89 \pm 0,14$} & \multicolumn{2}{|c|}{$0,88 \pm 0,16$} & \multicolumn{4}{|c|}{$0,61 \pm 0,19$} \\
\hline \multicolumn{13}{|c|}{ SAF-T-SIDE } \\
\hline 35 & $91,67 \mathrm{a}$ & $0,16 \pm 0,07$ & $\mathrm{R}$ & $91,67 \mathrm{a}$ & $0,16 \pm 0,07$ & $\mathrm{R}$ & $95,00 \mathrm{a}$ & $0,10 \pm 0,13$ & $\mathrm{R}$ & $95,00 \mathrm{a}$ & $0,10 \pm 0,22$ & $\mathrm{R}$ \\
\hline 10 & $83,33 \mathrm{ab}$ & $0,30 \pm 0,07$ & $\mathrm{R}$ & $86,67 \mathrm{ab}$ & $0,25 \pm 0,07$ & $\mathrm{R}$ & $90,00 \mathrm{a}$ & $0,19 \pm 0,00$ & $\mathrm{R}$ & $93,33 \mathrm{a}$ & $0,13 \pm 0,07$ & $\mathrm{R}$ \\
\hline 1 & $70,00 \mathrm{~b}$ & $0,49 \pm 0,13$ & $\mathrm{R}$ & $73,33 \mathrm{~b}$ & $0,44 \pm 0,07$ & $\mathrm{R}$ & $75,00 \mathrm{~b}$ & $0,41 \pm 0,13$ & $\mathrm{R}$ & $78,33 \mathrm{a}$ & $0,37 \pm 0,07$ & $\mathrm{R}$ \\
\hline 0,60 & $50,00 \mathrm{c}$ & $0,70 \pm 0,00$ & $\mathrm{R}$ & $53,33 \mathrm{c}$ & $0,66 \pm 0,15$ & $\mathrm{R}$ & $53,33 \mathrm{c}$ & $0,65 \pm 0,07$ & $\mathrm{R}$ & $56,67 \mathrm{~b}$ & $0,63 \pm 0,20$ & $\mathrm{R}$ \\
\hline 0,20 & $41,67 \mathrm{~cd}$ & $0,77 \pm 0,15$ & $\mathrm{R}$ & $43,33 \mathrm{~cd}$ & $0,75 \pm 0,15$ & $\mathrm{R}$ & $46,67 \mathrm{~cd}$ & $0,71 \pm 0,20$ & $\mathrm{R}$ & $46,67 \mathrm{~b}$ & $0,72 \pm 0,20$ & $\mathrm{R}$ \\
\hline 0,10 & $36,67 \mathrm{~cd}$ & $0,81 \pm 0,20$ & A & $38,33 \mathrm{~d}$ & $0,79 \pm 0,20$ & $\mathrm{R}$ & $40,00 \mathrm{~d}$ & $0,77 \pm 0,13$ & $\mathrm{R}$ & $41,67 \mathrm{~b}$ & $0,76 \pm 0,20$ & $\mathrm{R}$ \\
\hline 0,01 & $35,00 \mathrm{~d}$ & $0,82 \pm 0,22$ & $\mathrm{~A}$ & $36,67 d$ & $0,80 \pm 0,07$ & $\mathrm{R}$ & $36,67 d$ & $0,79 \pm 0,07$ & $\mathrm{R}$ & $41,67 \mathrm{~b}$ & $0,76 \pm 0,07$ & $\mathrm{R}$ \\
\hline Testigo & $6,67 \mathrm{e}$ & - & - & $5,00 \mathrm{e}$ & - & - & $3,33 \mathrm{e}$ & - & - & $5,00 \mathrm{c}$ & - & - \\
\hline $\operatorname{Pr}>X^{2}$ & \multicolumn{3}{|c|}{$<, 0001$} & \multicolumn{3}{|c|}{$<, 0001$} & \multicolumn{2}{|r|}{$<, 0001$} & \multicolumn{4}{|c|}{$<, 0001$} \\
\hline $\mathrm{X}^{2}$ & \multicolumn{3}{|c|}{36,90} & & 34,92 & & & 33,95 & & & 24,69 & \\
\hline $\mathrm{CR}_{50}$ & & $0,38(0,11-1,08)$ & & & $5(0,06-0,70)$ & & & $(0,04-0,49)$ & & & $4(0,02-0,49)$ & \\
\hline $\mathrm{b} \pm \mathrm{s}$ & & $0,53 \pm 0,09$ & & & $0,54 \pm 0,09$ & & & $, 58 \pm 0,10$ & & & $0,58 \pm 0,12$ & \\
\hline & & & & & STY & ET- & & & & & & \\
\hline 35 & $90,00 \mathrm{a}$ & $0,19 \pm 0,13$ & $\mathrm{R}$ & $91,67 \mathrm{a}$ & $0,16 \pm 0,15$ & $\mathrm{R}$ & $93,33 \mathrm{a}$ & $0,13 \pm 0,07$ & $\mathrm{R}$ & $91,67 \mathrm{a}$ & $0,16 \pm 0,07$ & $\mathrm{R}$ \\
\hline 10 & $58,33 \mathrm{~b}$ & $0,60 \pm 0,07$ & $\mathrm{R}$ & $61,67 \mathrm{~b}$ & $0,58 \pm 0,07$ & $\mathrm{R}$ & $63,33 \mathrm{~b}$ & $0,56 \pm 0,07$ & $\mathrm{R}$ & $61,67 \mathrm{~b}$ & $0,58 \pm 0,07$ & $\mathrm{R}$ \\
\hline 6 & $48,33 \mathrm{bc}$ & $0,70 \pm 0,07$ & $\mathrm{R}$ & $48,33 \mathrm{c}$ & $0,70 \pm 0,07$ & $\mathrm{R}$ & $51,67 \mathrm{c}$ & $0,68 \pm 0,07$ & $\mathrm{R}$ & $53,33 \mathrm{bc}$ & $0,67 \pm 0,07$ & $\mathrm{R}$ \\
\hline 2 & $41,67 \mathrm{~cd}$ & $0,75 \pm 0,07$ & $\mathrm{R}$ & $41,67 \mathrm{c}$ & $0,76 \pm 0,07$ & $\mathrm{R}$ & $43,33 \mathrm{~cd}$ & $0,76 \pm 0,07$ & $\mathrm{R}$ & $50,00 \mathrm{c} \mathrm{d}$ & $0,70 \pm 0,13$ & $\mathrm{R}$ \\
\hline 1 & $40,00 \mathrm{~cd}$ & $0,77 \pm 0,13$ & $\mathrm{R}$ & $38,33 \mathrm{c}$ & $0,79 \pm 0,07$ & $\mathrm{R}$ & $40,00 \mathrm{~d}$ & $0,78 \pm 0,13$ & $\mathrm{R}$ & $41,67 \mathrm{de}$ & $0,77 \pm 0,07$ & $\mathrm{R}$ \\
\hline 0,35 & $36,67 \mathrm{~d}$ & $0,79 \pm 0,15$ & $\mathrm{R}$ & $38,33 \mathrm{c}$ & $0,79 \pm 0,15$ & $\mathrm{R}$ & $36,67 \mathrm{~d}$ & $0,81 \pm 0,07$ & $\mathrm{R}$ & $33,33 \mathrm{e}$ & $0,83 \pm 0,07$ & $\mathrm{R}$ \\
\hline 0,10 & $16,67 \mathrm{e}$ & $0,93 \pm 0,07$ & $\mathrm{~N}$ & $16,67 \mathrm{~d}$ & $0,93 \pm 0,13$ & $\mathrm{~A}$ & $16,67 \mathrm{e}$ & $0,94 \pm 0,07$ & A & $13,33 \mathrm{f}$ & $0,96 \pm 0,07$ & $\mathrm{~A}$ \\
\hline Testigo & $3,33 \mathrm{f}$ & - & - & $5,00 \mathrm{~d}$ & - & - & $6,67 \mathrm{f}$ & - & - & $6,67 \mathrm{f}$ & - & - \\
\hline $\operatorname{Pr}>X^{2}$ & & $<, 0001$ & & & $<, 0001$ & & & $<, 0001$ & & & $<, 0001$ & \\
\hline $\mathrm{X}^{2}$ & & 25,98 & & & 24,79 & & & 32,59 & & & 43,41 & \\
\hline $\mathrm{CR}_{50}$ & & $3,31(1,32-10,84)$ & & & $(1,34-11,64)$ & & & $4(1,51-8,90)$ & & & $2(1,70-7,34)$ & \\
\hline $\mathrm{b} \pm \mathrm{s}$ & & $0,69 \pm 0,13$ & & & $0,73 \pm 0,15$ & & & $, 79 \pm 0,14$ & & & $0,82 \pm 0,12$ & \\
\hline
\end{tabular}

${ }^{1}$ Rep: Repelencia; ${ }^{2}$ IR: Índice de repelencia; ${ }^{3}$ Clasificación: R: Repelente; N: Neutral; A = Atrayente; ${ }^{4}$ Intervalos de confianza a $95 \%$. b: Pendiente de la línea de regresión. s: Error estándar. ${ }^{5}$ Medias con distinta letra en una columna son estadísticamente diferentes (Tukey $\mathrm{p} \leq 0,05$ ). $\mathrm{n}=800$ insectos tratados.

Los cuatro aceites ocasionaron una inhibición de la oviposición. A la concentración de $1 \mathrm{mg} / \mathrm{mL}$ se observó una reducción de la oviposición $\geq 93,63 \%$; el comportamiento fue similar en los cuatro aceites evaluados $(\mathrm{p}<0,05)$ (Tabla 2). La concentración inhibitoria media $\left(\mathrm{CIO}_{50}\right)$ en los cuatro productos evaluados osciló entre 0,0006 y $0,003 \mathrm{mg} / \mathrm{mL}$ y el valor de la pendiente en todos los casos fue menor a la unidad, lo cual indica respuesta heterogénea de los adultos. Anasef-T fue el aceite que mostró el mejor efecto inhibitorio hasta dosis de 0,001 mg/mL (Tabla 2). 
Tabla 2. Inhibición de oviposición (\%) de Diaphorina citri a las 48 h después de la aplicación de cuatro aceites minerales.

\begin{tabular}{|c|c|c|c|c|}
\hline \multirow{2}{*}{$\begin{array}{l}\text { Concentración } \\
(\mathrm{mg} / \mathrm{mL})\end{array}$} & AKAROIL & ANASEF-T & SAF-T-SIDE & STYLET-OIL \\
\hline & \multicolumn{4}{|c|}{ Inhibición (\%) } \\
\hline 1,00 & 93,63 a1 & 94,67 a & $94,67 \mathrm{a}$ & 96,08 a \\
\hline 0,35 & $77,71 \mathrm{ab}$ & - & - & - \\
\hline 0,1 & $73,89 \mathrm{bc}$ & $90,00 \mathrm{a}$ & $74,00 \mathrm{~b}$ & $77,78 \mathrm{~b}$ \\
\hline 0,035 & $63,69 \mathrm{bcd}$ & - & $68,67 \mathrm{~b}$ & $69,93 \mathrm{~b}$ \\
\hline 0,01 & $58,60 \mathrm{~cd}$ & $84,67 \mathrm{ab}$ & $67,33 \mathrm{~b}$ & $67,32 \mathrm{cb}$ \\
\hline 0,0035 & - & $75,33 \mathrm{bc}$ & $50,00 \mathrm{c}$ & - \\
\hline 0,001 & $52,23 \mathrm{de}$ & $66,00 \mathrm{c}$ & $41,33 \mathrm{c}$ & $49,67 \mathrm{~cd}$ \\
\hline 0,00035 & - & - & $25,33 \mathrm{~d}$ & - \\
\hline 0,0001 & $36,31 \mathrm{e}$ & $48,67 \mathrm{~d}$ & - & $35,29 \mathrm{de}$ \\
\hline 0,000035 & - & $28,67 \mathrm{e}$ & - & - \\
\hline 0,00001 & - & - & - & $31,37 \mathrm{e}$ \\
\hline Testigo & $0,00 \mathrm{f}$ & $0,00 \mathrm{f}$ & $0,00 \mathrm{e}$ & $0,00 \mathrm{f}$ \\
\hline $\mathrm{N}$ & 800 & 800 & 800 & 800 \\
\hline $\operatorname{Pr}>X^{2}$ & $<, 0001$ & $<, 0001$ & $<, 0001$ & $<, 0001$ \\
\hline $\mathrm{X}^{2}$ & 26,62 & 39,89 & 36,48 & 39,34 \\
\hline $\mathrm{CIO}_{50}$ & $0,001(0,0002-0,004)$ & $0,0002(0,00005-0,0005)$ & $0,003(0,001-0,007)$ & $0,0006(0,0001-0,002)$ \\
\hline $\mathrm{b} \pm \mathrm{s}$ & $0,36 \pm 0,07$ & $0,50 \pm 0,08$ & $0,57 \pm 0,09$ & $0,38 \pm 0,06$ \\
\hline
\end{tabular}

b: Pendiente de la línea de regresión; s: error estándar; - Dosis no evaluada; ${ }^{1}$ Medias con distinta letra en la misma columna son estadísticamente diferentes (Tukey $\mathrm{p} \leq 0,05$ ).

Para ambos ensayos se observó que existe una relación directa entre la concentración utilizada y la respuesta; es decir, a mayor concentración mayor es el efecto repelente e inhibitorio de la oviposición, aunque el grado de actividad depende del producto evaluado (Tabla 3). Como se puede observar en la Tabla 3 la aplicación de dosis de $1 \mathrm{mg} / \mathrm{mL}$, con todos los aceites, es suficiente para inhibir en más del $90 \%$ la oviposición, en cambio sí se requiere causar un efecto repelente similar se necesitan aplicar dosis de $35 \mathrm{mg} / \mathrm{mL}$.

Tabla 3. Relación repelencia e inhibición de oviposición (\%) de Diaphorina citri a las 24 y $48 \mathrm{~h}$, respectivamente, después de la aplicación de cuatro aceites minerales.

\begin{tabular}{lllllllll}
\hline \multirow{2}{*}{$\begin{array}{c}\text { Concen. } \\
\mathbf{m g} / \mathbf{m L}\end{array}$} & \multicolumn{2}{c}{ AKAROIL } & \multicolumn{2}{c}{ ANASEF } & \multicolumn{2}{c}{ SAF-T-SIDE } & \multicolumn{2}{c}{ STYLET-OIL } \\
\cline { 2 - 10 } & Rep. $^{1}$ & IO $^{2}$ & Rep. & IO & Rep. & IO & Rep. & IO \\
\hline 35,00 & 96,6 & - & 96,6 & - & 91,6 & - & 95,0 & - \\
\hline 10,00 & 81,6 & - & 81,6 & - & 61,6 & - & 93,3 & - \\
\hline 6,00 & 61,6 & - & 65,0 & - & 53,3 & - & - & - \\
\hline 1,00 & 33,3 & 93,6 & 33,3 & 94.6 & 41,6 & 94,6 & 78,3 & 96,0 \\
\hline 0,35 & - & 77,7 & 26,6 & - & 33,3 & - & - & - \\
\hline 0,10 & 21,6 & 73,8 & 26,6 & 90.0 & 13,3 & 74,0 & 41,6 & 77,7 \\
\hline 0,035 & - & 63,6 & - & - & - & 68,6 & - & 69,9 \\
\hline 0,01 & 11,6 & 58,6 & - & 84.6 & - & 67,3 & 41,6 & 67,3 \\
\hline 0,001 & - & 52,2 & - & 66.0 & - & 41,3 & & 49,6 \\
\hline 0,0001 & - & 36,3 & - & 48.6 & - & - & & 35,2 \\
\hline Testigo & 5,0 & 00,0 & 3,3 & 00.0 & 6,6 & 0,0 & 5,0 & 0,0 \\
\hline
\end{tabular}

${ }^{1}$ Rep: Repelencia; ${ }^{2} \mathrm{IO}$ : Inhibición de Oviposición; - Dosis no evaluada.

\section{Discusión}

Los resultados obtenidos en este estudio muestran que los aceites minerales evaluados tienen la capacidad de repeler a adultos e inhibir la oviposición de $D$. citri, hallazgo que coincide con lo obtenido por diversos autores para el control de diversas plagas asociadas a los cítricos (Rae et al. 1997; Chen et al. 2009; Hoy 2011). El alto nivel de repelencia obtenido por los aceites evaluados se atribuye, entre otros, a la persistencia; característica relacionada con la viscosidad y la baja volatilidad de los aceites (Najar-Rodríguez et al. 2008). El grado de viscosidad y densidad de los aceites los hace más pesados y por ello son retenidos por más tiempo sobre la hoja, de modo que las sustancias repelentes se liberan gradualmente, retrasando o evitando el arribo de los insectos a la planta tratada (Najar-Rodríguez et al. 2008; Stadler y Buteler 2009). Repelencia similar (>59\%) a la encontrada en este estudio, reportan varios autores al aplicar diferentes aceites como Caltex ${ }^{\circledR}$, Enspray 99, y D-C-Tron Plus (Leong et al. 2012; Ouyang et al. 2013; Yang et al. 2013) y, se asemejan a los obtenidos en el caso de Stylet-Oil ${ }^{\circledR}$ a dicha concentración, sin embargo, difieren de los obtenidos por Poerwanto et al. (2012) al aplicar Sunspray UltraFine $\AA$ y Enspray $99 \AA$ a $2 \%$; ellos obtuvieron repelencia de $61 \%$ y $57 \%$, respectivamente, en condiciones de laboratorio. El efecto repelente e inhibitorio de la oviposición de los aceites minerales se relaciona con la formación de una fina película sobre los tejidos de las plantas, después de la aplicación, lo cual interfiere con la emisión de volátiles liberados por las plantas, lo cual dificulta el reconocimiento del huésped por el insecto fitófago (Ouyang et al. 2008; Poerwanto et al. 2012). Ouyang et al. (2013) también señalaron que la respuesta diferencial en repelencia y deterrencia en oviposición causada por los aceites se relaciona con 
los porcentajes de masa y distribución de carbonos en la molécula, pero no con los emulsificantes en la formulación; por consiguiente, la correcta selección del aceite, combinación de dosis y cobertura adecuadas podría propiciar niveles de repelencia óptimos. Al parecer la mayor efectividad para reducir las poblaciones de $D$. citri e incidencia de HLB, en condiciones de campo, se logra con aceites cuya molécula contiene C22, C23, y C24, debido a que estos tienen un tamaño óptimo que les permite entrar por las estomas y bloquear la liberación de volátiles de la planta. Sin embargo, y debido a que los aceites actúan de manera moderada, en comparación con un insecticida convencional, debido a su rápida degradación en el ambiente, es importante aplicar los aceites de manera frecuente (cada 9 días), justo antes de la época de brotación (Rae et al. 1997; Ouyang et al. 2013). Además, el hecho de que los aceites presenten actividad repelente y/o inhibitoria de la oviposición sobre adultos de PAC permite inferir que el uso adecuado de éstos (composición, dosis, cobertura, velocidad de aplicación, etc.) coadyuvaría a retrasar y reducir el riesgo de diseminación del HLB, debido a que los aceites, al alterar el comportamiento de búsqueda de huéspedes, pueden reducir o retrasar el arribo y contacto de la población de insectos vectores, y con ello disminuir la alimentación y adquisición de la bacteria y por tanto el número de vectores virulíferos, lo que implica una baja en la tasa de transmisión (Leong et al. 2012; Vidal et al. 2012; Hu et al. 2020). Aunado a lo anterior, Rae et al. (1997) y Ouyang et al. (2013) señalaron que los aceites son una alternativa viable para el manejo del psílido, por ser compatibles con otras opciones de bajo riesgo, como feromonas, aceites vegetales, jabones, hongos entomopatógenos, depredadores y parasitoides, lo que permitiría su incorporación a un programa de Manejo Integrado de Plagas.

La efectividad de los aceites, como se observó en este estudio, depende de la concentración; pero también el aumento de ésta incrementa el riesgo de fitotoxicidad razón por la cual es importante la aplicación de las concentraciones efectivas. En este caso particular, se puede lograr un buen efecto repelente e inhibitorio de la oviposición de $D$. citri con la aplicación de los aceites a concentraciones de $10 \mathrm{mg} / \mathrm{ml}$ (equivalente a la aplicación en campo a una concentración de $1 \%$ $\mathrm{v} / \mathrm{v})$. Recomendable también es el evitar la aplicación de los aceites en ambientes con temperaturas superiores a $35{ }^{\circ} \mathrm{C}$ o por debajo de $5^{\circ} \mathrm{C}$ y en condiciones de sequía, sobre plantas muy debilitadas por ataques de plagas o con deficiente estado nutricional (Stadler y Buteler 2009; Buteler y Stadler 2011).

En este contexto se sugiere se realicen estudios profundos, en condiciones de campo, con el fin de obtener información sobre la efectividad de los aceites al interaccionar con los diversos factores prevalecientes para determinar si éstos pueden constituirse en un componente potencial en el manejo integrado de D. citri.

\section{Conclusiones}

Los aceites minerales tienen la capacidad de repeler a adultos e inhibir la oviposición de D. citri. El efecto repelente tuvo una relación positiva al aumento de la concentración. Las concentraciones de $35 \mathrm{mg} / \mathrm{mL}$ en todos los productos evaluados consiguieron una repelencia $\geq 90 \%$. Dosis de $1 \mathrm{mg} / \mathrm{mL}$, con todos los aceites, fueron suficientes para inhibir hasta en $90 \%$ la oviposición. Por tanto, se infiere que los aceites minerales podrían representar una herramienta útil en campo para disminuir y retrasar el arribo de $D$. citri en plantaciones de cítricos.

\section{Literatura citada}

ABBOTT, W. S. 1925. A method of computing the effectiveness of an insecticide. Journal of Economic Entomology 18 (2): 265267. https://doi.org/10.1093/jee/18.2.265a

BEATTIE, G. A.; LIU, Z. M.; WATSON, D. M.; CLIFT, A. D.; JIANG, L. 1995. Evaluation of petroleum spray oils and polysaccharides for control of Phyllocnistis citrella Stainton (Lepidoptera: Gracillariidae). Journal Australian Entomology Society 34: 349-353. https://doi.org/10.1111/j.1440-6055.1995.tb01353.x

BUTELER M.; STADLER, T. 2011. A review on the mode of action and current use of petroleum distilled spray oils, p. 119-136. En: Management. Stoytcheva, M. (Ed.). Pesticides in the modern World - Pesticides use. https://doi.org/10.5772/20394

CHEN, C.; ZHENG, J.; XIE, J.; XIE, X.; MAO, R. 2009. Pest management based on petroleum spray oil in navel orange orchard in Ganzhou, south China. Journal of Pest Science 82: 155-162. https://doi.org/10.1007/s10340-008-0234-9

DA GRAÇA, J.; DOUHAN, G.; HALBERT, S.; KEREMANE, M.; LEE, R..; VIDALAKIS, G.; ZHAO, H. 2016. Huanglongbing: an overview of a complex pathosystem ravaging the world's citrus. Journal of Integrative Plant Biology 58 (4), 373-387. https:// doi.org/10.1111/jipb.12437

DEL PUERTO, R. A.; SUÁREZ, S. T.; PALACIO, E. D. 2014. Efectos de los plaguicidas sobre el ambiente y la salud. Revista Cubana de Higiene y Epidemiología 52 (3): 372-387. http://scielo.sld.cu/ scielo.php?script $=$ sci_arttext\&pid=S1561-30032014000300010

GARCÍA-MÉNDEZ, V. H.; ORTEGA-ARENAS, L. D.; VILLANUEVA-JIMÉNEZ, J. A.; SÁNCHEZ-ARROYO, H. 2016. Susceptibilidad de Diaphorina citri Kuwayama (Hemiptera: Liviidae) a insecticidas en Veracruz, México. Agrociencia 50: 355-365. http://www.redalyc.org/articulo.oa?id=30246007008.

GARCÍA-MÉNDEZ, V. H.; ORTEGA-ARENAS, L. D.; VILLANUEVA-JIMÉNEZ, J.A.; OSORIO-ACOSTA, F. 2018. Resistencia de Diaphorina citri Kuwayama a insecticidas en cinco Áreas Regionales de Control en México. Southwestern Entomologist 44 (4): 947-954. https://doi.org/10.3958/059.044.0415

HERNÁNDEZ-FUENTES, L. M., URIAS-LÓPEZ, M. A., LÓPEZ-ARROYO, J. I., GÓMEZ-JAIMES, R., BAUTISTA-MARTÍNEZ, N. 2012. Control químico de Diaphorina citri Kuwayama (Hemiptera: Psyllidae) en lima Persa Citrus latifolia Tanaka. Revista Mexicana de Ciencias Agrícolas 3 (3): 427-439. https://doi.org/10.29312/remexca.v3i3.1439

HOY, M. A. 2011. Agricultural Acarology: Introduction to Integrated Mite Management. New York. CRC Press. 410 p.

HU, W.; ZHENG, R.; FENG, X.; KUANG, F.; CHUN, J.; XU, X.; CHEN, T.; LU, J.; LI, W.; ZHANG, N. 2020. Emergence inhibition, repellent activity and antifeedant responds of mineral oils against Asian citrus psyllid, Diaphorina citri (Hemiptera: Liviidae), International Journal of Pest Management Ahead of print 1-8. https://doi.org/10.1080/09670874.2020.1847354.

KALLIANPUR, A. S.; HERRON, G. A.; BEATTIE, G. A.; WATSON, D. M. 2002. Potter sprays tower bioassays of two horticultural mineral oils against tomato thrips, tomato russet mite and greenhouse whitefly adults, and common brown leafhopper nymphs. p. 112-117. En: Beattie, G. A.; Watson, D. M.; Stevens, M. L.; Rae, D. J.; Spooner-Hart, R. N. (Eds.). Spray Oils Beyond 2000 - Sustainable Pest and Disease Management. University of Western Sydney. Australia. 627 p.

LEONG, S. C.; ABANG, F.; BEATTIE, G. A.; KUEH, R. J.; WONG, S. K. 2012. Impacts of horticultural mineral oils and two insecticide practices on population fluctuation of Diaphorina citri and spread of Huanglongbing in a citrus orchard in Sarawak. The Scientific World Journal 2012 : 651416. https:// doi.org/10.1100/2012/651416

MARTÍNEZ-FERRER, M. T.; FIBLA, J. M.; CAMPOS, J. M.; BELTRÁN, E.; RIPOLLÉS, J. L. 2003. Aplicación de aceites minerales insecticidas en árboles adultos de cítricos para el control de Phyllocnistis citrella (Lepidoptera: Gracillaridae) y otras plagas de verano (I): Eficacia sobre plagas. Boletín de Sanidad Vegetal 
Plagas 29: 281-289. https://www.miteco.gob.es/ministerio/pags/ Biblioteca/Revistas/pdf_plagas\%2FBSVP-29-02-281-289.pdf

MORA-AGUILERA, G.; ROBLES-GARCÍA, P.; LÓPEZ-ARROYO, J. I.; FLORES-SÁNCHEZ, J.; ACEVEDO-SÁNCHEZ, G.; DOMÍNGUEZ-MONGE, S.; GUTIÉRREZ-ESPINOSA, A.; LOEZA-KUK, E.; GONZÁLEZ-GÓMEZ, R. 2014. Situación actual y perspectivas de manejo del HLB de los cítricos. Revista Mexicana de Fitopatología 32 (2): 108-119. http://www.scielo.org.mx/scielo.php?script=sci_arttext\&pi$\mathrm{d}=\mathrm{S} 0185-33092014000200108$

NAJAR-RODRÍGUEZ, A. J.; LAVIDIS, N. A.; MENSAH, R. K.; CHOY, P. T.; WALTER, G. H. 2008. The toxicological effects of petroleum spray oils on insects-evidence for an alternative mode of action and possible new control options. Food and Chemical Toxicology 46 (9): 3003-3014. https://doi.org/10.1016/j. fct.2008.05.042

NGUYEN, V. L.; MEATS, A.; BEATTIE, G. A.; SPOONER-HART, R.; LIU, Z. M.; JIANG, L. 2007. Behavioral responses of female Queensland fruit fly, Bactrocera tryoni, to mineral oil deposits. Entomologia Experimentalis et Applicata 122 (3): 215-221. https://doi.org/10.1111/j.1570-7458.2006.00504.x

OLVERA-VARGAS, L. A., QUIROZ GASPAR, A. J., CONTRERAS-MEDINA, D. I., AGUILAR-RIVERA, N. 2020. Análisis de riesgo potencial de Huanglongbing a través de tecnología geoespacial en Colombia. Ciencia y Tecnología Agropecuaria 21 (3): e1552. https://doi.org/10.21930/rcta.vol21_num3_art:1552

OUYANG, G. C.; YANG, Y. P.; ZHONG, G. L.; XIONG, J. J.; HUANG, M. D.; LIANG, G. W. 2008. Oviposition repellency and EAG responses the oriental fruit fly, Bactrocera dorsalis (Hendel) to mineral spray oils. Acta Entomologica Sinica 51 (4): 390-394. http://www.insect.org.cn/EN/Y2008/V51/I4/390

OUYANG, G. C.; FANG, X.; LU, H.; ZHOU, X.; MENG, X.; YU, S.; GUO, M.; XIA, Y. 2013. Repellency of five mineral oils against Diaphorina citri (Hemiptera: Liviidae). Florida Entomologist 96 (3): 974-982. https://doi.org/10.1653/024.096.0335

PÉREZ-ZARATE, L. A.; OSORIO-ACOSTA, F.; VILLANUEVA-JIMÉNEZ, J. A.; ORTEGA-ARENAS, L. D.; CHIQUITO-CONTRERAS, R. G. 2016. Factores que inciden en el control químico de Diaphorina citri Kuwayama en áreas regionales de control. Southwestern Entomologist 41 (4): 1037-1050. http:// doi.org/10.3958/059.041.0404

POERWANTO, M. E.; TRISYONO, Y. A.; BEATTIE, G. A. C.; SUBANDIYAH, S.; MARTONO, E.; HOLFORD, P. 2012. Olfactory response of Asiatic citrus psyllid (Diaphorina citri) to mineral oil treated mandarin leaves. Journal of Nature Studies 10 (2): 32-37. https://doi.org/10.3844/ajabssp.2012.50.55

RAE, D. J.; LIANG, W. G.; WATSON, D. M.; BEATTIE, G. A. C.; HUANG, M. D. 1997. Evaluation of petroleum spray oils for control of the Asian citrus psylla, Diaphorina citri (Kuwayama) (Hemiptera: Psyllidae) in China. International Journal of Pest Management 43 (1): 71-75. http://doi.org/10.1080/096708797229013

ROBERTSON, J. L.; RUSSELL, R. M.; PREISLER, H. K.; SAVIN, E. 2007. Bioassays with Arthropods. CRC Press, Boca Raton, FL, U.S.A. 2nd edition, xxii + 199 p. https://doi. org/10.1201/9781420004045

SAS (Statistical Analysis System). 2004. SAS/STAT 9.1 User's Guide. SAS Publishing. Cary, North Carolina. 5180 p. https://www. support.sas.com/.../cdl/en/statugstatmodel/.../statugstatmodel

SCHUSTER, D. J.; THOMPSON, S.; ORTEGAA., L. D.; POLSTON, J. E. 2009. Laboratory evaluation of products to reduce settling of sweetpotato whitefly adults. Journal of Economic Entomology 102 (4): 1482-1489. https://doi.org/10.1603/029.102.0412

SENASICA. 2020. Manual operativo de la campaña contra plagas de los cítricos. V1. 71 p. https://www.gob.mx/cms/uploads/attach-
ment/file/614759/Manual_operativo_Plagas_de_los_C_tricos. pdf [Fecha de revisión: 25 agosto de 2021].

SIEBURTH, P. J.; SCHROEDER, W. J.; MAYER, R. T. 1998. Effects of oil and oil-surfactant combinations on silverleaf whitefly nymphs (Homoptera: Aleyrodidae) on collards. Florida Entomologist 81 (3): 446-450. https://doi.org/10.2307/3495935

SMITH, E. H.; SALKELD, E. H. 1966. The use and action of ovicides. Annual Review of Entomology 11: 331-368. https://doi. org/10.1146/annurev.en.11.010166.001555

STADLER, T.; BUTELER, M. 2009. Modes of entry of petroleum distilled spray-oils into insects: a review. Bulletin of Insectology 62 (2): 169-177. https://ri.conicet.gov.ar/handle/11336/81261

STANSLY, P. A.; LIU, T. X.; SCHUSTER, D. J. 2002. Effects of horticultural mineral oils on a polyphagous whitefly, its plant hosts and its natural enemies. pp. 120-133. En: Beattie, G. A. C.; Watson, D. M.; Stevens, M. L.; Rae, D. J.; Spooner-Hart, R. N. (Eds.). Spray oils beyond 2000 - Sustainable pest and disease management. University of Western Sydney, Australia. 627 p.

TAVERNER, P. 2002. Drowning or just waving? A perspective on the ways petroleum-derived oils kill arthropod pests of plants, pp. 78-87. En: Beattie, G. A. C.; Watson, D. M.; Stevens, M. L.; Rae, D. J.; Spooner-Hart, R. N. (Eds.). Spray oils beyond 2000 Sustainable pest and disease management. University of Western Sydney, Australia. 627 p.

TIWARI, S.; STELINSKI, L. L.; ROGERS, M. E. 2012. Biochemical basis of organophosphate and carbamate resistance in Asian citrus psyllid. Journal of Economic Entomology 105 (2): 540548. http://doi.org/10.1603/EC11228

VARELA, B.; CABALEIRO C.; LÓPEZ, M. 2004. Empleo de aceites de distinto origen, en programas de manejo integrado en pimiento para el control del pulgón Myzus persicae (Sulzer) Boletín de Sanidad Vegetal Plagas 30: 185-195. https://www.miteco. gob.es/ministerio/pags/Biblioteca/Revistas/pdf_plagas\%2FBSVP-30-01-02-185-195.pdf

VIDAL, E.; MARTÍNEZ, M.; BERTOLINI, E.; CAMBRA, M. 2012. Aceites minerales para el control de virus de vegetales. Aplicación a Plum pox virus en viveros. Phytoma España 241: 1-7. https://www.phytoma.com/la-revista/phytohemeroteca/242-octubre-2012/aceites-minerales-para-el-control-de-virus-de-vegetales-aplicacin-a-plum-pox-virus-en-viveros

YANG, Y.; BEATTIE, G. A.; SPOONER-HART, R.; HUANG, M.; BARCHIA, I.; HOLFORD, P. 2013. Influences of leaf age and type, non-host volatiles, and mineral oil deposits on the incidence, distribution, and form of stylet tracks of Diaphorina citri. Entomologia Experimentalis et Applicata 147: 33-49. https://doi. org/10.1111/eea.12046

\section{Origen y financiación}

El presente artículo derivó del trabajo de grado del estudiante Mario Apáez Barrios del Programa en Entomología y Acarología- Fitosanidad del Colegio de Postgraduados.

\section{Contribuciones de los autores}

El primer y segundo autor diseñaron y ejecutaron el experimento, colectaron los datos, hicieron los análisis estadísticos y redactaron el manuscrito. Todos los autores participaron en la supervisión, revisión y edición del manuscrito.

\section{Conflictos de interés}

Los autores declaramos no tener conflictos de intereses. 